\title{
NUMERICAL SIMULATION OF METAL MELT FLOWS IN MOLD CAVITY WITH CERAMIC POROUS MEDIA
}

\author{
CHANGCHUN DONG, "JIANXIN ZHOU, YAJUN YIN, ZHAO GUO \\ State Key Laboratory of Materials Processing and Die \& Mould Technology, \\ Huazhong University of Science \& Technology, Wuhan 430074, Hubei, China \\ \#E-mail: zhoujianxin@hust.edu.cn
}

Submitted September 23, 2015; accepted February 2, 2016

\begin{abstract}
Keywords: Numerical simulation, Metal matrix composites, Flow process, Porous media, Ceramic preform
Process modeling of metal melt flow in porous media plays an important role in casting of metal matrix composites. In this work, a mathematical model of the metal melt flow in preform ceramic particles was used to simulate the flow behavior in a mold cavity. The effects of fluid viscosity and permeability (mainly affected by porosity of ceramic preforms) on the flow behavior were analyzed. The results indicate that ceramic porous media have a significant effect on the flow behavior by contributing to a low filling velocity and sharp pressure drop in the cavity. The pressure drop has a linear relationship with the fluid velocity, and a nonlinear relationship with porosity. When the porosity is relatively small, the pressure drop is extremely large. When porosity exceeds a certain value, the pressure drop is independent of porosity. The relationship between viscosity and porosity is described, and it is shown that the critical porosity changes when the viscosity of the melt changes. However, due to the limited viscosity change, the critical porosity changes by less than 0.043.
\end{abstract}

\section{INTRODUCTION}

Metal matrix composites (MMCs) have received increasing attention in recent years due to their relatively high elastic modulus, tensile strength, temperature stability and wear resistance [1,2]. These mechanical properties give MMCs potential for applications in the automotive, medical and aerospace fields. MMCs are primarily reinforced using fibers, whiskers or particles. Porous ceramic preforms are one type of particle reinforcement used in MMCs, and play a paramount role among various types of MMCs [3,4]. The dispersion strengthening properties of ceramics give these MMCs better resistance, tensile strength and dimensional stability relative to the parent metal, and higher fracture toughness compared to parent ceramics.

Infiltration routes are commonly used in the manufacturing of MMCs [5, 6]. During the manufacturing process, liquid metal is pushed through a preformed porous ceramic by an external pressure, yielding MMCs after solidification. The infiltration process has a direct effect on the formation and quality of MMCs. Therefore it is crucial to study the flow of immiscible fluids in porous media. Numerical simulation is the most commonly used method for mathematical modeling of multiphase flows [7].

Currently, research on fluid flow in porous media has attracted extensive interest. The reactivity of polymers has been studied by Liu et al. [8] by simulating the fluid flow in heterogeneous porous media, but, of course, the reactivity makes the simulation conditions more complicated. Chang et al. [9] found that the flow rate decreases exponentially with increasing infiltration time. Moreover, they also studied infiltration time in various boundary conditions. Sampath et al. [10] simulated unidirectional flow in squeeze casting. They concluded that the three main factors affecting the infiltration were the liquid superheating temperature, the preform preheating temperature and the inlet pressure. Jung et al. [11] simulated the infiltration of liquid steel into the porous region, with coupled simulation of temperature fields. This simulation yielded reasonable inlet pressures and mold preheating temperatures. Klostermann et al. [12] investigated the infiltration of liquid steel into porous ceramic structures at the pore-size scale of the porous structures, and obtained both temperature fields and flow fields. Liu et al. [13] calculated the flow rate of argon gas into the liquid steel by using a three-dimensional porousflow model. The model determined the flow distribution and estimated the initial mean bubble size in the nozzle. Preform infiltration is an alternative of the casting process, but both share the common characteristic of fluid flow, which is necessary to enable flow of the metal melt in the porous ceramic.

In this work, numerical simulation was used to comprehensively investigate fluid flow in a mold cavity with a porous ceramic preform. Unlike previous models, this simulation is implemented by using a specially designed geometry. It aims at explaining the basic principle of the flow process and the mechanism of infiltration in the specified cavity with porous media. 


\section{THEORETICAL}

Physical basis assumptions

The multiphase model used in this work is the socalled "Volume of Fluid" (VOF) model. The VOF model is carried out in two phases: (1) metal melt, (2) gas. There are three components (phase regions) in the system: (1) metal melt, (2) gas, and (3) ceramic particles. Since it is impossible to establish the ceramic particles geometry in detail and generate the meshes, the area where the ceramic particles located is treated as a porous zone. The fluid flow in the porous zone is exposed to a much more powerful resistance than in air. The viscosity of metal melt is mainly dependent on two factors: temperature and alloy elements. In general, viscosity decreases with increasing temperature. From this, the following assumptions are proposed:

a) The viscosity of the metal melt is between $1.5 \mathrm{mPa} \cdot \mathrm{s}$ and $2.5 \mathrm{mPa} \cdot \mathrm{s}$ [14].

b) The velocity of the gas is kept at a low level, so that both the gas and the liquid metal are incompressible.

c) Capillary pressure is negligible when compared with the infiltration pressure.

d) The ceramic porous preform is isotropic. The porous medium momentum resistance is calculated separately for each phase.

\section{Governing equations}

Common equations used to solve fluid flow are the continuity equation and Navier-Stokes equations [15]. From the above assumptions, both the gas and the liquid metal are incompressible, and since the density of both fluids is constant, the continuity equations can be written as

$$
\operatorname{div} \boldsymbol{u}=0,
$$

where $\boldsymbol{u}$ is the fluid velocity.

The Navier-Stokes equations can be written as follows:

$$
\begin{aligned}
& \frac{\partial(\rho u)}{\partial t}+\operatorname{div}(\rho u \mathbf{u})=\operatorname{div}(\mu \operatorname{grad} u)-\frac{\partial p}{\partial x}+\rho g_{x}+S_{x}, \\
& \frac{\partial(\rho v)}{\partial t}+\operatorname{div}(\rho v \mathbf{u})=\operatorname{div}(\mu \operatorname{grad} v)-\frac{\partial p}{\partial y}+\rho g_{y}+S_{y}, \\
& \frac{\partial(\rho w)}{\partial t}+\operatorname{div}(\rho w \mathbf{u})=\operatorname{div}(\mu \operatorname{grad} w)-\frac{\partial p}{\partial z}+\rho g_{z}+S_{z},
\end{aligned}
$$

where $u, v$ and $w$ is the velocity for the $x, y$ and $z$ direction, respectively, $\mu$ is the dynamic viscosity of the fluid, $g_{x}$, $g_{y}$ and $g_{z}$ is the gravity acceleration for the $x, y$ and $z$ direction, respectively, and $S_{x}, S_{y}$ and $S_{z}$ is the source term for the $x, y$ and $z$ component of the Navier-Stokes equations, respectively.

In this study, porous media are modeled by the addition of a momentum source term to the standard
Navier-Stokes equations (see Equation 2, the NavierStokes equations without $S$ term are the standard NavierStokes equations). The source term is composed of two parts: a viscous loss term, $\sum_{j=1}^{3} D_{i j} \mu u_{j}$ and an inertial loss term, $\sum_{j=1}^{3} C_{i j} \frac{1}{2} \rho|u| u_{j}$, i.e.

$$
\begin{aligned}
& S_{x}=\sum_{j=1}^{3} D_{x j} \mu u_{j}+\sum_{j=1}^{3} C_{x j} \frac{1}{2} \rho|u| u_{j}, \\
& S_{y}=\sum_{j=1}^{3} D_{y j} \mu u_{j}+\sum_{j=1}^{3} C_{y j} \frac{1}{2} \rho|u| u_{j}, \\
& S_{z}=\sum_{j=1}^{3} D_{z j} \mu u_{j}+\sum_{j=1}^{3} C_{z j} \frac{1}{2} \rho|u| u_{j},
\end{aligned}
$$

where $|\mathrm{u}|$ is the magnitude of the velocity, $D_{i j}$ is a matrix related to the permeability of the ceramic preform, these two quantities being reciprocal to each other, i.e. $D_{i j}=$ $=1 / k_{i j}$, and $C_{i j}$ is the inertia resistance factor, a constant that can be viewed as a loss coefficient per unit length along flow direction.

Unlike fiber reinforcement, isometric ceramic particles usually have no preferential direction. Therefore, ceramic particle porous media can usually be considered as isotropic porous media, and this Equation 3 can be simplified as (see Appendix A)

$$
\begin{aligned}
& S_{x}=\frac{\mu}{k} u+C \frac{1}{2} \rho|u| u, \\
& S_{y}=\frac{\mu}{k} v+C \frac{1}{2} \rho|u| v, \\
& S_{z}=\frac{\mu}{k} w+C \frac{1}{2} \rho|u| w,
\end{aligned}
$$

where, $k$ is the permeability of the ceramic preform. The value of permeability can be written as Equation 5 [16] and $C$ can be expressed as Equation 6 [17]:

$$
\begin{gathered}
k=\frac{\varepsilon^{5 / 2} r_{p}^{2}}{8(\ln \varepsilon)^{2}}, \\
C=\frac{3.5}{d_{p}} \frac{1-\varepsilon}{\varepsilon^{3}},
\end{gathered}
$$

where, $\varepsilon$ is the porosity of the ceramic preform, $r_{p}$ is the radius of the pores and $d_{p}$ is the mean particle diameter of the ceramic preform.

\section{EXPERIMENTAL}

\section{Calculation parameters}

In order to observe the effect of porous media on the flow field, a geometric model with a porous medium in the middle area has been created (Figure 1). Figure 1a) is a three-dimensional geometric model, which contains an inlet, outlet, porous medium zone, while the rest of 
the region represents the cavity. In the actual production, the porous medium zone in Figure 1a usually represents the ceramic preform particles. This geometric model can be used to model the flow process involved in the manufacturing of MMCs. Metal melt is injected from the inlet, then liquid metal flows through the cavity. The flow process is finished when the cavity is completely filled. Due to the axisymmetric characteristics of this 3-D model, a longitudinal section is used to demonstrate the geometric size of the 3-D model, as depicted in Figure 1b. The diameters of the inlet and outlet are both $50 \mathrm{~mm}$ while the diameters of cavity and ceramic preform are both $150 \mathrm{~mm}$. The length of every section is also labeled in the figure.

The axisymmetric model is used in this calculation, and the mesh of the model is shown in Figure 2. The size of the mesh is $2.5 \mathrm{~mm} \times 2.5 \mathrm{~mm}$, and the total number of

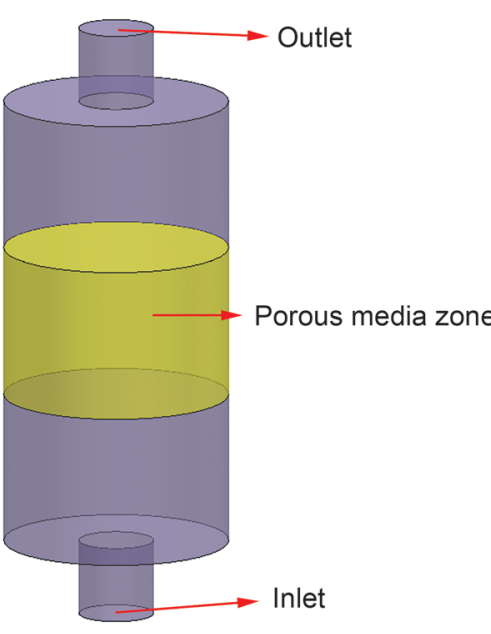

a)

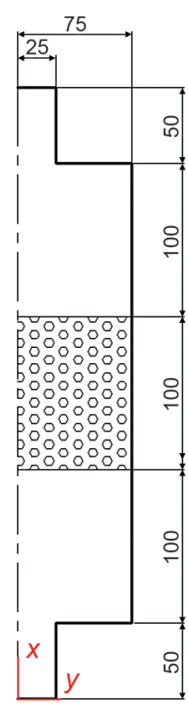

b)
Figure 1. Schematic of 3-D geometry (a) and main dimensions (b).

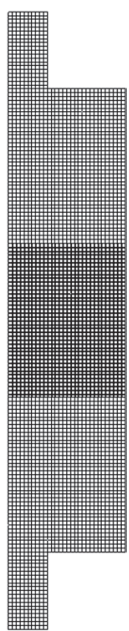

Figure 2. Mesh figure of the calculation model: normal lines = default cavity zone; bold lines = porous medium zone. the mesh is 4000 . In the following numerical simulation, both transient and steady flows were performed to observe flow behaviors in the calculation domains. The commercial CFD code ANSYS Fluent was used to implement the flow simulation in the porous media. The velocity and pressure gradients of fluid flow were calculated in the porous medium. The magnitudes of viscosity and different porosity were also considered in fluid flow simulation.

\section{Numerical experiments}

\section{Transient flow in the mold cavity}

A multiphase flow in the cavity was simulated. The model was applied to an infiltration-casting processing,

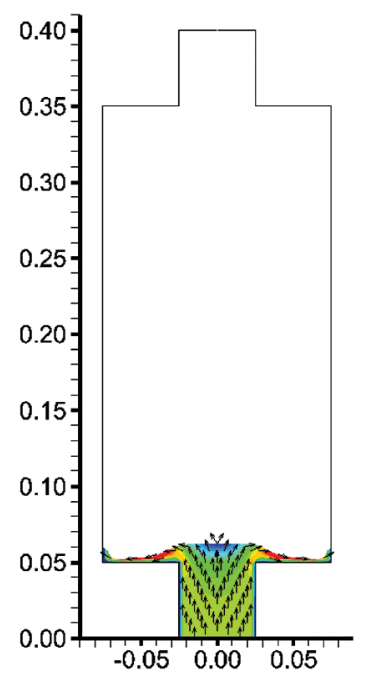

a)

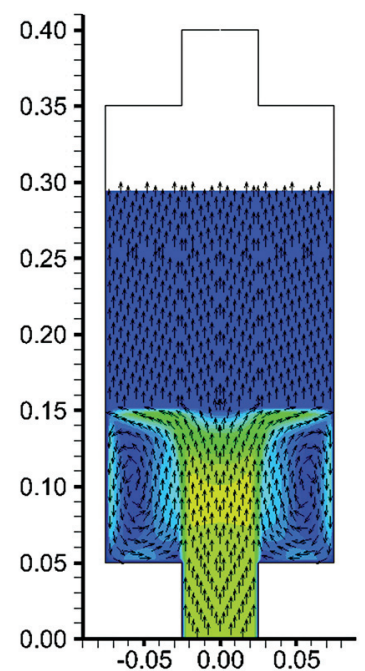

c)

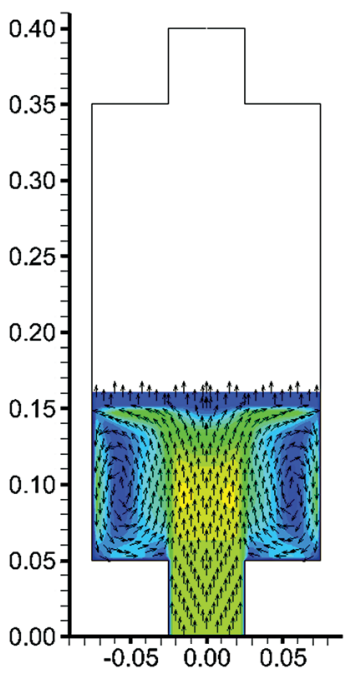

b)

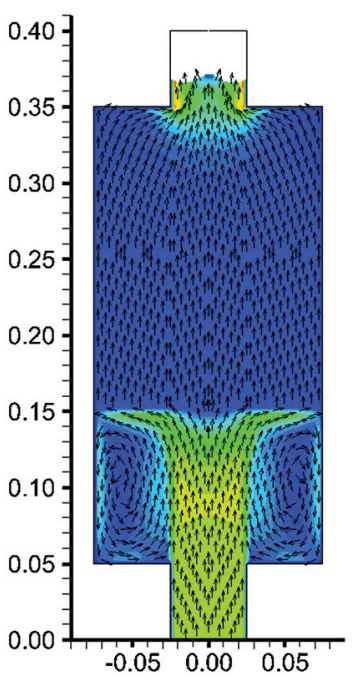

d)
Figure 3. Flow behavior in the mold cavity: a) $t=0.5 \mathrm{~s}$, b) $\mathrm{t}=5.0 \mathrm{~s}, \mathrm{c}) \mathrm{t}=9.0 \mathrm{~s}, \mathrm{~d}) \mathrm{t}=11.5 \mathrm{~s}$. 
where inlet velocity $=0.2 \mathrm{~m} \cdot \mathrm{s}^{-1}$, outlet pressure $=0 \mathrm{~Pa}$ (absolute pressure minus atmospheric pressure is $0 \mathrm{~Pa}$ ), porosity $=0.5$ and gravity $=9.8 \mathrm{~m} \cdot \mathrm{s}^{-2}$. Figure 3 shows the casting process of the model containing the porous media and the velocity distribution of the liquid metal. Before the metal melt reached the ceramic preforms, the flow front had tiny fluctuations and was not flat. When the infiltration started, the flow front reached the porous medium. Due to the viscous loss caused by the porous medium, the flow front became very flat.

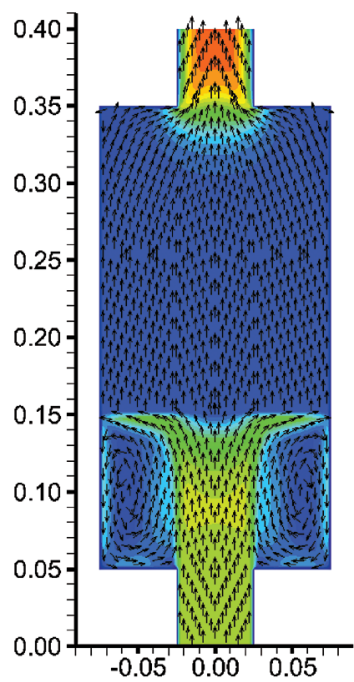

a)

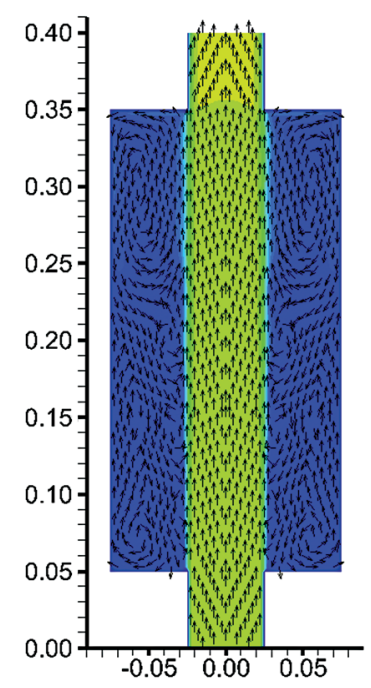

b)

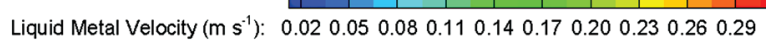

Figure 4. Velocity in the cavity: a) porosity $=0.5$, b) porosity $=1.0$.

\section{Velocity and pressure in the mold cavity}

Figure $4 \mathrm{a}$ illustrates the fluid velocity vector when the mold cavity is fulfilled with liquid metal. It shows that a vortex formed between inner wall of the mold and wall of the ceramic preform that at the mold inlet side. The formation of vortices can be explained as follows: since the mold cavity is filled with liquid metal, the fluid flows from the inlet with an external pressure comes into contact with the ceramic porous medium. Due to the blocking effect of the ceramic porous medium, a portion of the fluid infiltrated the porous medium, and the rest flows to the inner wall of the mold. The fluid portion that flows to the inner wall of the mold then falls down by its own weight. When it reaches the inner wall of the mold at the bottom, it meets the fluid from the mold inlet, and then they flow forward together. As a result, vortices are formed. There is no porous medium in the mold cavity when the porosity $=1.0$. As can be seen in Figure $4 b$, in this case the vortex formed in the top of the cavity. Comparing these two conditions, we found that the porous medium can greatly change the flow behavior in the cavity. For this specific example, the porous medium changed the fluid fields and moved the vortex from the top of the cavity to the bottom of the cavity.

Figure 5 shows the pressure distribution in the cavity at a steady state. In the porous zone, the pressure dropped sharply. The pressure drop was mainly attributed to the viscous loss of the fluid caused by the porous structure. Figure 5 also shows the detailed pressure distribution of the rectangular area. When compared with the porous medium, the pressure drop in the rectangle area was very low. This is expected because the fluid flow had much lower resistance in air than that in the porous medium. However, the pressure distribution in the rectangular area is not uniform, and experiences a pressure drop.
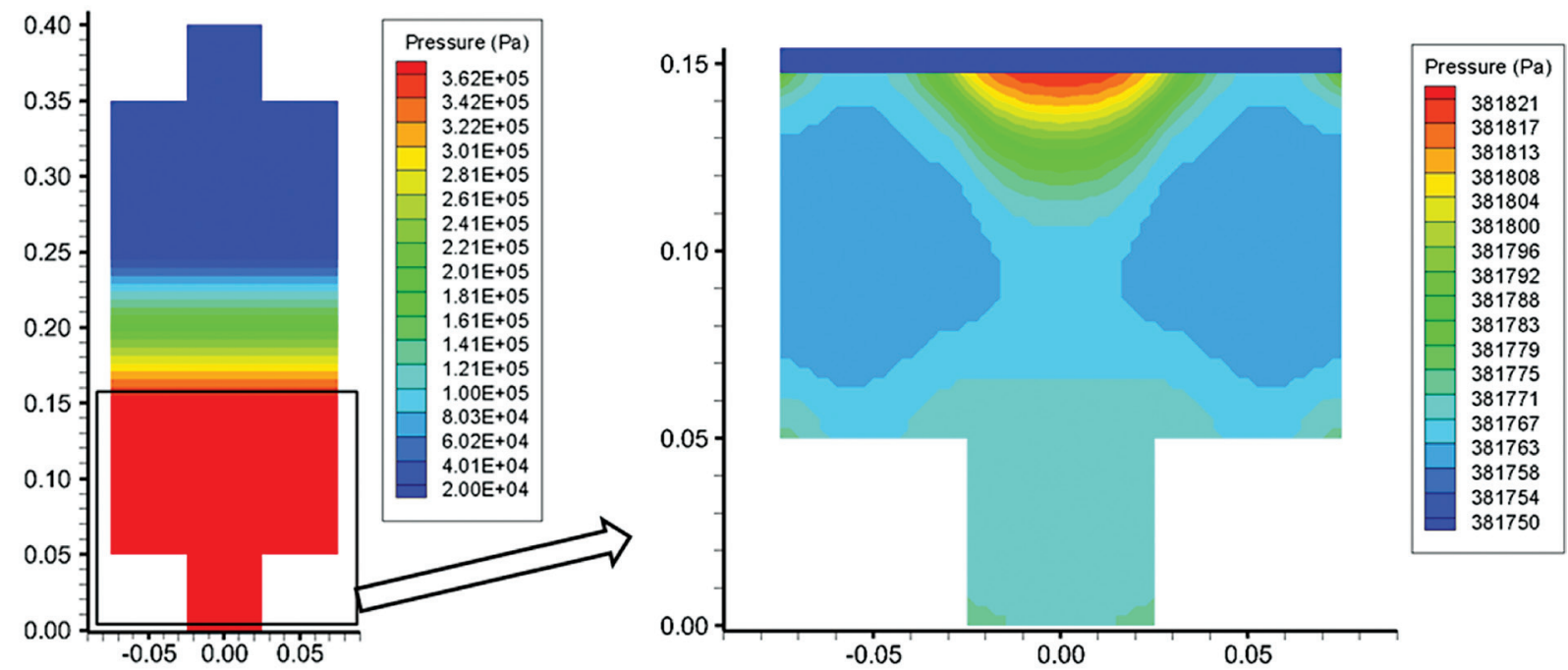

Figure 5. Contours of static pressure. 
Figure 6 shows the velocity magnitude along the model axis. The velocity curve can be divided into three sections:

1) section between the mold cavity inlet and the ceramic porous preform, $x=0 \mathrm{~m} \sim 0.15 \mathrm{~m}$,

2) section in the porous medium, $x=0.15 \mathrm{~m} \sim 0.25 \mathrm{~m}$,

3 ) section between preform and mold cavity outlet, $x=$ $0.25 \mathrm{~m} \sim 0.40 \mathrm{~m}$.

In the area between the inlet and the porous preform, the velocity is $0.2 \mathrm{~m} \cdot \mathrm{s}^{-1}$ in the inlet area, and after the fluid flows into the cavity from the inlet, the velocity slowly increased to $0.21 \mathrm{~m} \cdot \mathrm{s}^{-1}$ at $\mathrm{x}=0.1 \mathrm{~m}$. Then, the velocity dropped sharply to about $0.025 \mathrm{~m} \cdot \mathrm{s}^{-1}$ at $x=0.15 \mathrm{~m}$. In the porous medium area, the velocity maintained a low level. After the fluid flows out of the porous medium, the velocity increases to about $0.27 \mathrm{~m} \cdot \mathrm{s}^{-1}$ at the outlet.

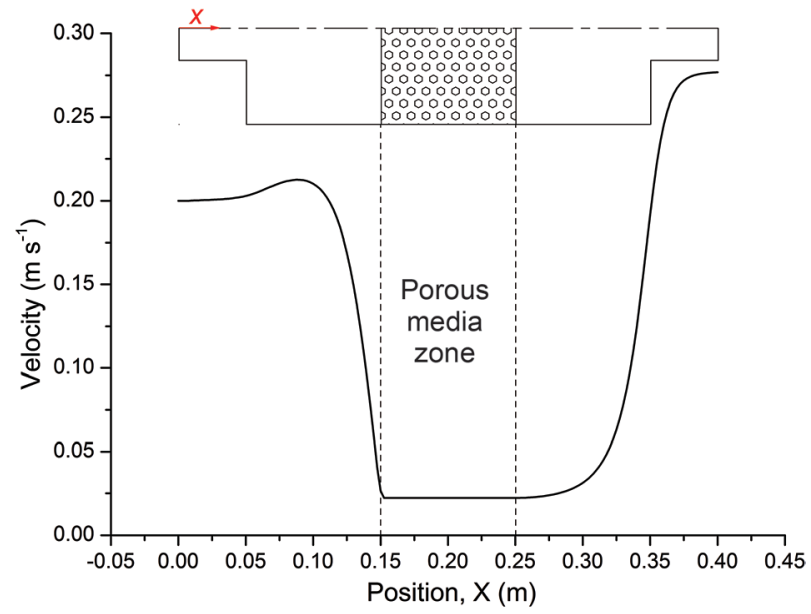

Figure 6. Velocity along $\mathrm{x}$-axis.

\section{Effect of fluid viscosity}

In order to study the effects of viscosity on fluid flow in a cavity, pressure distributions with various fluid viscosities were analyzed. As depicted in Figure 7,

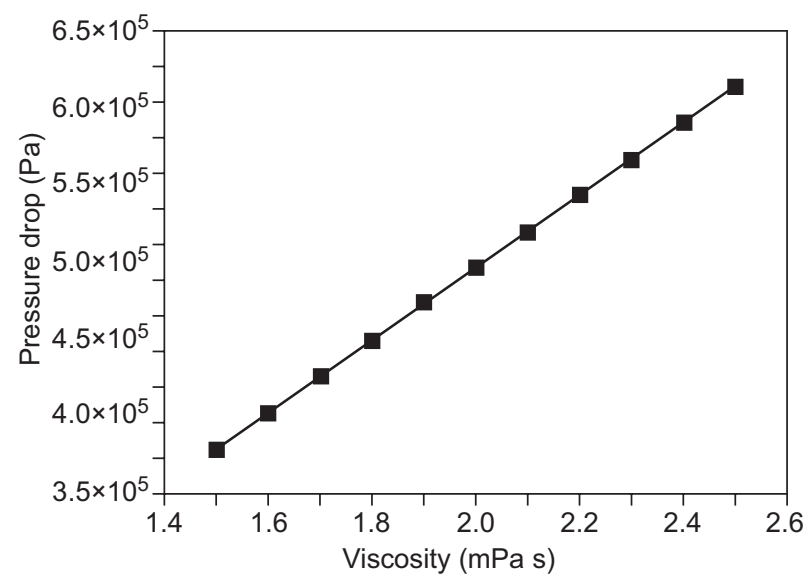

Figure 7. Pressure drop with various fluid viscosities. pressure drop had a linear relationship with fluid viscosity. When the viscosity of the metal melt was $1.5 \mathrm{mPa} \cdot \mathrm{s}$, the corresponding pressure drop was about $3.82 \times 10^{5} \mathrm{~Pa}$; when the viscosity of the metal melt was $2.5 \mathrm{mPa} \cdot \mathrm{s}$, the corresponding pressure drop was about $6.36 \times 10^{5} \mathrm{~Pa}$. So some measures may be taken to optimize the process parameters. For example, the fluid viscosity can be changed by changing the metal melt temperature or adding some elements to the metal melt, and thus the specific external pressure can be adopted to accomplish the manufacturing of MMCs.

\section{Effect of porosity}

In this paper, porosity was defined as the percentage of void space in the porous ceramic preform. Using the simulation model, the effect of permeability on the flow process was calculated. Porosity played the primary role in permeability. Therefore ceramic preforms with porosities from 0.2 to 0.9 were simulated, and the relationship between the pressure drop and porosity was calculated, as shown in Figure 8.

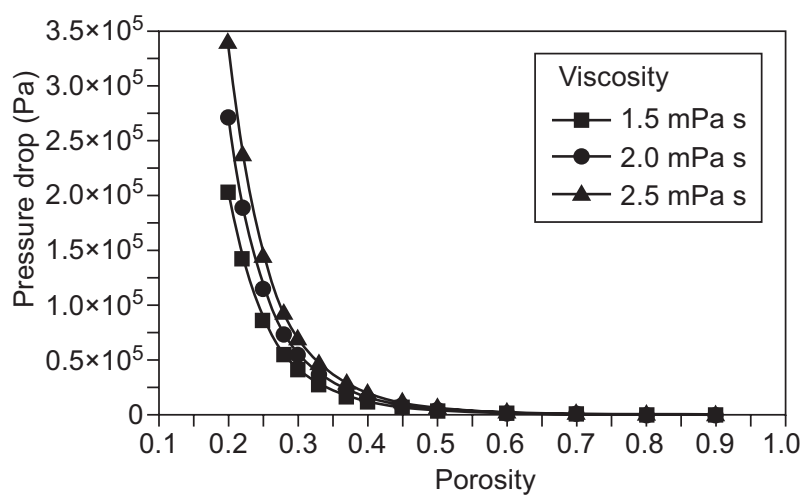

Figure 8. Pressure drop with various porosity and viscosity.

When the porosity was less than $0.3 \sim 0.4$, the pressure drop decreased sharply with increasing porosity (Figure 8), But when the porosity was more than $0.3 \sim 0.4$, the pressure drop changed only slightly with increasing porosity. The lower the porosity, the more difficult is metal melt flow in the porous medium. Higher porosity results in less reinforcement in the MMCs, which reduces the overall mechanical performance of the MMCs. As a result, it is necessary to have an optimal porosity to guarantee both the infiltration quality and the final properties.

Different porosities were also simulated in combination with different viscosities $(1.5 \mathrm{mPa} \cdot \mathrm{s}, 2.0 \mathrm{mPa} \cdot \mathrm{s}$ and $2.5 \mathrm{mPa} \cdot \mathrm{s}$ ), see Figure 8 . Under the specific pressure drop, different viscosity corresponded to various porosity (for example, if the pressure drop is $5.0 \times 10^{6} \mathrm{~Pa}$, the fluids with viscosity of $1.5 \mathrm{mPa} \cdot \mathrm{s}, 2.0 \mathrm{mPa} \cdot \mathrm{s}$ and $2.5 \mathrm{mPa} \cdot \mathrm{s}$ corresponding to porosity of $0.289,0.310$ and 0.323 respectively). The critical porosity, defined as the 
porosity below which the fluid cannot flow through the porous zone, is important to make an appropriate casting process. Of course, the critical porosity is higher when the viscosity is higher, And thus the critical porosity changes when the viscosity is changed. In this study, under the same pressure drop of the three viscosities, their critical porosity varied from 0 to 0.043 .

\section{CONCLUSIONS}

In this study, a mathematical model was established to simulate the fluid flow process in cavities with porous media. A simple geometry was chosen for applying this mathematical model. Factors taken into consideration in the model included the viscosity of the fluid and the porosity of the ceramic preform. The model predicts the pressure gradient for different viscosities and porosities. The following conclusions can be drawn from the study:

- When metal melt fills the cavity, the frontier becomes very flat in the porous zone. In the steady state simulation, the pressure drops sharply in the porous zone under low flow velocity.

- The pressure gradient is directly proportional to the fluid viscosity, the relationship between the two being linear. Therefore, modifying the metal melt viscosity is an efficient tool to control the external pressure.

- The porosity of the ceramic preform has a significant effect on the pressure gradient. When the porosity is less than 0.3 , the relationship between the two is exponential. When the porosity is low, the pressure drop is large. When the porosity is above 0.4 , the pressure drop changes only slightly with increasing porosity. Thus, from the simulation result it is recommended that the porosity of ceramic preform should not be less than 0.3 .

- As far as the critical porosity in this work is concerned, for different viscosities of the liquid metal, the value of the critical porosity is different.

\section{Acknowledgements}

This research was financially supported by State Major Science and Technology Special Project Foundation for High-end Numerical Machine and Basic Manufacturing Equipment (2012ZX04012-011) and key project of State Key Laboratory of Materials Processing and Die \& Mould Technology (2015).

\section{Appendix A. Derivation of Equation 4 as implemented in the software}

The porous media model incorporates an empirically determined flow resistance in a region of our model defined as "porous". In essence, the porous media model is nothing more than an added momentum sink in the governing momentum equations. As such, the following modeling assumptions should be readily recognized as follows (Porous Media Conditions. ANSYS 13.0 Help, Fluent 7.2.3.):

- Since the volume blockage that is physically present is not represented in the model, by default ANSYS FLUENT uses and reports a superficial velocity inside the porous medium, based on the volumetric flow rate, to ensure continuity of the velocity vectors across the porous medium interface.

- The porous media momentum resistance and heat source terms are calculated separately on each phase.

- For the ceramic preform, since the shape of the ceramic particles is assumed to be spherical or near spherical. For the simplification of the problems, the porous medium is considered as isotropic in this work.

In general, when the ceramic preform is assumed to be anisotropic, Equations (3) can be written as

$$
\begin{aligned}
& \left(\begin{array}{c}
S_{x} \\
S_{y} \\
S_{z}
\end{array}\right)=\mu\left(\begin{array}{lll}
D_{11} & D_{12} & D_{13} \\
D_{21} & D_{22} & D_{23} \\
D_{31} & D_{32} & D_{33}
\end{array}\right)\left(\begin{array}{l}
u \\
v \\
w
\end{array}\right)+ \\
& +\frac{1}{2} \rho \mid u\left(\left(\begin{array}{lll}
C_{11} & C_{12} & C_{13} \\
C_{21} & C_{22} & C_{23} \\
C_{31} & C_{32} & C_{33}
\end{array}\right)\left(\begin{array}{l}
u \\
v \\
w
\end{array}\right)\right.
\end{aligned}
$$

When the porous medium is assumed to be isotropic, this equation is simplified via the conditions

$D_{12}=D_{13}=D_{23}=D_{21}=D_{31}=D_{32}=0, D_{11}=D_{22}=D_{33}=$ $=1 / k ; C_{12}=C_{13}=C_{23}=C_{21}=C_{31}=C_{32}=0, C_{11}=C_{22}=$ $=C_{33}=C$

and Equation $\mathrm{S} 1$ can be simplified as

$$
\begin{aligned}
& \left(\begin{array}{l}
S_{x} \\
S_{y} \\
S_{z}
\end{array}\right)=\mu\left(\begin{array}{ccc}
1 / k & 0 & 0 \\
0 & 1 / k & 0 \\
0 & 0 & 1 / k
\end{array}\right)\left(\begin{array}{l}
u \\
v \\
w
\end{array}\right)+ \\
& +\frac{1}{2} \rho|u|\left(\begin{array}{ccc}
C & 0 & 0 \\
0 & C & 0 \\
0 & 0 & C
\end{array}\right)\left(\begin{array}{l}
u \\
v \\
w
\end{array}\right)
\end{aligned}
$$

Equation S2 is just Equation 4 in the main text.

\section{REFERENCES}

1. Schobel M., Requena G., Fiedler G., Tolnai D., Vaucher S., Degischer H. P. (2014): Void formation in metal matrix composites by solidification and shrinkage of an AlSi7 matrix between densely packed particles. Composites Part a-Applied Science and Manufacturing, 66, 103-108. doi:10.1016/j.compositesa.2014.07.011

2. Mishra S. K., Satapathy A. (2014): Ceramic particulate filled ZA-27 metal matrix composites: comparative analysis. Materials Science and Technology, 30, 1495-1499. doi:10.1179/1743284713Y.0000000453 
3. Lemster K., Graule T., Kuebler J. (2005): Processing and microstructure of metal matrix composites prepared by pressureless Ti-activated infiltration using $\mathrm{Fe}$-base and $\mathrm{Ni}$ base alloys. Materials Science and Engineering a-Structural Materials Properties Microstructure and Processing, 393, 229-238. doi:10.1016/j.msea.2004.10.025

4. Lemster K., Delporte A., Graule T., Kuebler J. (2007): Activation of alumina foams for fabricating MMCs by pressureless infiltration. Ceramics International, 33, 11791185. doi:10.1016/j.ceramint.2006.04.002

5. Skirl S., Krause R., Wiederhorn S. M., Rodel J. (2001): Processing and mechanical properties of $\mathrm{Al}_{2} \mathrm{O}_{3} / \mathrm{Ni}_{3} \mathrm{Al}$ composites with interpenetrating network microstructure. Journal of the American Ceramic Society, 84, 2034-2040. doi:10.1111/j.1151-2916.2001.tb00954.x

6. Akhtar F. (2014): Ceramic reinforced high modulus steel composites: processing, microstructure and properties. Canadian Metallurgical Quarterly, 53, 253-263. doi:10.11 79/1879139514Y.0000000135

7. Allen M. B. (1985): Numerical modelling of multiphase flow in porous media. Advances in Water Resources, 8, 162-187.

8. Liu H. G., Thompson K. E. (2002): Numerical modeling of reactive polymer flow in porous media. Computers \& Chemical Engineering, 26, 1595-1610. doi:10.1016/S00981354(02)00130-8

9. Chang C. Y. (2006): Numerical simulation of the pressure infiltration of fibrous preforms during MMC processing. Advanced Composite Materials, 15, 287-300. doi:10.1163/ 156855106778392070

10. Sampath V., Ramanan N., Palaninathan R. (2006): Modeling of liquid metal infiltration of porous fiber preform during squeeze casting. Materials and Manufacturing Processes, 21, 495-505. doi:10.1080/10426910500471524

11. Jung C. K., Jang J. H., Han K. S. (2008): Numerical simulation of infiltration and solidification processes for squeeze cast al composites with parametric study. Metallurgical and Materials Transactions a-Physical Metallurgy and Materials Science, 39A, 2736-2748. doi:10.1007/s11661008-9619-x

12. Klostermann J., Schwarze R., Weider M., Brucker C. (2011): Computational Fluid Dynamic (CFD) Simulations of Liquid Steel Infiltration in Ceramic Foam Structures. Part II: Application to Laboratory-Scale Experiments. Steel Research International, 82, 1113-1121. doi:10.1002/ srin.201100093

13. Liu R., Thomas B. G., Sengupta J. (2012) in: Mcwasp Xiii: International Conference on Modeling of Casting, Welding and Advanced Solidification Processes, 1-10. doi:10.1088/1757-899X/33/1/011001

14. Sun Y. S. (2013). The Viscosity Research of Al-Fe Alloys. Master, Shandong University, JiNan, ShanDong, China.

15. Versteeg H. K., Malalasekera W. (2007). An Introduction to Computational Fluid Dynamics: The Finite Volume Method. $2^{\text {nd }}$ ed. Prentice Hall.

16. Xu P. (2013). Transient simulation of the densification process in isothermal chemical vapor infiltration of thickwalled carbon/carbon composites. Doctor, Huazhong University of Science and Technology, Wuhan, China.

17. Ergun S. (1952): Flow through Packed Columns. Chemical Engineering Progress, 48, 89-94. 